\title{
3D numerical study of Hill mounted VAWT
}

\author{
Belabes Belkacem \\ Centre de Développement des Energies Renouvelables, \\ CDER, BP 62 Route de l'Observatoire, Bouzaréah, 16340 \\ Algiers, Algeria \\ belabes.belkacem@gmail.com
}

\author{
Marius Paraschivoiu \\ Concordia University, Montréal, H3G1M8, Québec, \\ Canada \\ marius.paraschivoiu@concordia.ca
}

\begin{abstract}
The ground topography effect on the wind flow is significant. The knowledge of the flow behavior near ground is crucial in the development of wind power, especially in the choice of suitable sites and for estimation of energy production. In this paper, the numerical prediction of the flow over a three-dimensional hill model and the analysis of placement of Savonius turbines on top of the hill are presented. The numerical analysis is based on the finite volume method implemented in the ANSYS CFX 15 Software using the Shear-Stress Transport (SST) turbulence model. The numerical results for a conventional Savonius rotor and a vertical-axis spiral wind rotor are both satisfactory compared with experimental data. The performances of these turbines, installed on the hilltop, are studied for different height positions. Furthermore, the influence of the hill size on the extracted power is investigated. At $\mathrm{TSR}=1$, the power coefficient of a conventional rotor is increased from 0.15 to 0.32 when the rotor is installed at a height of $0.25 \mathrm{~m}$ above the top of the hill, while it reaches 0.40 when the hill is two to three times higher. The helical Savonius rotor tested gives even higher power coefficient of 0.44 .
\end{abstract}

Keywords-component; Savonius wind turbine; 3D Computational Fluid Dynamics; SST turbulence model; Power Coefficient; hill mounted turbine

\section{INTRODUCTION}

The ground topography affects the velocity, direction and turbulence intensity of the wind and can be used advantageously to increase energy extraction from wind. In this work, a hill topology is considered. Flow separation at the lee side of a hill is still an unresolved issue where the field measurements show a rapid decrease in speed-up ratio. Different explanations are available regarding the issue; for instance: [1] conjectured that this three dimensional flow separation at the lee slope is due to the blockage effect of neighboring hills, but according to [2] the presence of the nearby topography does not affect the flow neither at the top nor at the downstream of the hill. For [2], hills have a significant influence on the wind speed. A smooth and not too steep hill causes acceleration of the wind and makes the wind accelerate when flowing towards the hilltop. The resultant increase in energy content is called hill impact. [2] and [3], find a good agreement between the experimental results and the computational results both at windward slope and at the lee side of the hill. [4] Investigate two-dimensional numerical simulations of flow over two common types of topographic features (a cliff and a hill) and three types of turbulence models. The standard k- $-\varepsilon$, Durbin's model and Shin's model are tested for predicting flow over this topographic. In this study, Shin's model gave better results compared to other models.

The wind flow over terrains can provide an important advantage when installing wind turbines. An appropriate selection of a suitable land for wind turbines can lead to an increased output of energy. However, understanding the many factors to be taken into account and the interaction with the turbine is needed. In this study, the interaction of the flow over the hill and the helical Savonius vertical axial wind turbine is analysed to increase the energy extraction.

The main objective of this paper is to analysis the improvement of the power output when the helical Savonius wind rotor developed by [5] and [6] are placed on top of a hill. The paper will show that a significant improvement of the power coefficient $(C p)$ can be achieved. In this study, the Savonius rotor is placed at different vertical locations on top of the hill and for different heights of the hill.

\section{HILL AND SAVONIUS WIND TURBINE GEOMETRIES}

The hill geometry employed in this work is shown in Fig. 1. It is a three-dimensional axisymmetric defined by [7] as follows:

$$
\mathrm{z}(\mathrm{x}, \mathrm{y})=\frac{\mathrm{H} \cos ^{2} \pi\left(\mathrm{x}^{2}+\mathrm{y}^{2}\right)^{\frac{1}{2}}}{2 \mathrm{~S}}
$$


The specific height used in the comparison with experimental data is $\mathrm{H}=40 \mathrm{~mm}$ and length $\mathrm{S}=100 \mathrm{~mm}$. It gives a maximum slope of about $32^{\circ}$. For the turbine mounted simulations, $\mathrm{H}=2 \mathrm{~m}$ and $\mathrm{S}=5 \mathrm{~m}$. Fig. 1 shows the details of the side view of the three dimensional hill and the coordinate system.

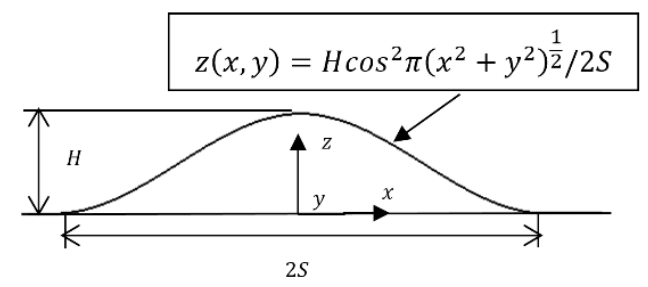

Figure 1. Topographic features [7]

The placement of turbines in complex land topology is becoming more and more common, although these areas are not always ideal sites, due to the high shear and turbulence levels in the wind flow. In this context, for a systematic analysis, the Savonius rotor is placed at a distance (L) above the hill. The Savonius rotor has an "S-shaped" cross-section constructed by two semicircular buckets. [6] conducted experimental investigations on a single stage helical Savonius rotor placed between end plates but without shaft. The helical rotor has a $90^{\circ}$ twist angle of the two blades evenly distributed around the vertical axis. The three-dimensional geometrical model of each blade can be approximately created by sweeping a base profile at $90^{\circ}$ along a spirally ascending curve, as shown in Fig. 2 and Table 1.
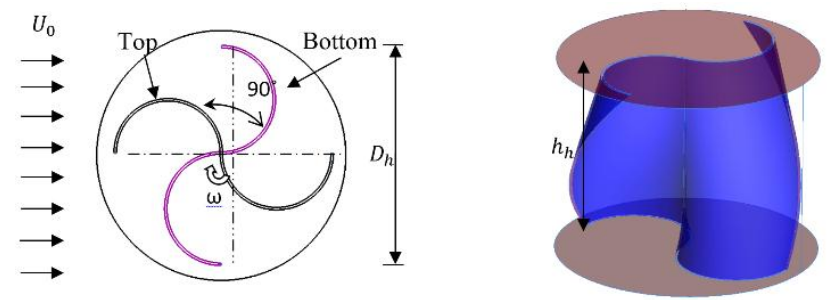

Figure 2. Schematic representations for a helical Savonius rotor .

TABLE I. BASIC PARAMETERS OF THE CONVENTIONAL SAVONIUS ROTOR [5] [6].

\begin{tabular}{|c|c|}
\hline Parameter & Value $(\mathrm{m})$ \\
\hline $\mathrm{D}$ & 0.33 \\
\hline $\mathrm{d}$ & 0.184 \\
\hline $\mathrm{e}$ & 0.038 \\
\hline $\mathrm{h}_{\mathrm{r}}$ & 0.23 \\
\hline $\mathrm{D}_{\mathrm{h}}$ & 0.23 \\
\hline $\mathrm{h}_{\mathrm{h}}$ & 0.202 \\
\hline
\end{tabular}

\section{ANALYSIS PARAMETERS}

Savonius turbines are mainly drag-driven devices made up of two or multiple blades. The difference in the drag forces acting on the rotor blades causes the Savonius turbine to rotate. The performance is defined by [5] as the power coefficient that is a function of power $\mathrm{P}$, rotor swept area $A s$, and free stream speed $U_{0}$ as follows:

$C_{P}=\frac{P}{\frac{1}{2} \rho A_{S} U_{0}^{3}}$

The moment coefficient $(\mathrm{Cm})$ of a Savonius turbine is determined as a function of torque $T$. It is defined by [5] as follows:

$C_{m}=\frac{T}{\frac{1}{4} \rho A_{s} D U_{0}^{2}}$

The Tip Speed Ratio TSR or $\lambda$ is the ratio between the rotational speed at the tip of the blade and the velocity of the wind. It is defined by [5] as follows:

$\lambda=\frac{\omega D}{2 U_{0}}$

The Reynolds number, $R e$, based on the rotor diameter is calculated as follows:

$R_{e}=\frac{\rho U_{0} D}{\mu}$

where $\mu$ is the dynamic viscosity of atmospheric air.

\section{NUMERICAL SIMULATION}

A hexahedral mesh is used for the stationary domain as shown in Fig. 3, and a tetrahedral mesh is used for the rotating domain containing the turbine as shown in Fig. 4. The total number of grid cells equals to 1.5106 . Note that the number of cells for both the conventional and helical rotors is almost the same. The meshes are generated using the ICEM CFD code. The grid node density in the rotating domain is higher than in the stationary domain. The prism layer of grid elements were extruded from the edges of the blade and the surface of the hill to improve the grid quality and to capture with sufficient precision the boundary layer flow. The height of the first prism layer above the surface was set such that the $y+$ value for the first layer of elements from the wall is below 2, depending on the rotation velocity of the rotor. There were nine layers in total, and a layer growth rate of 1.2 was chosen. 


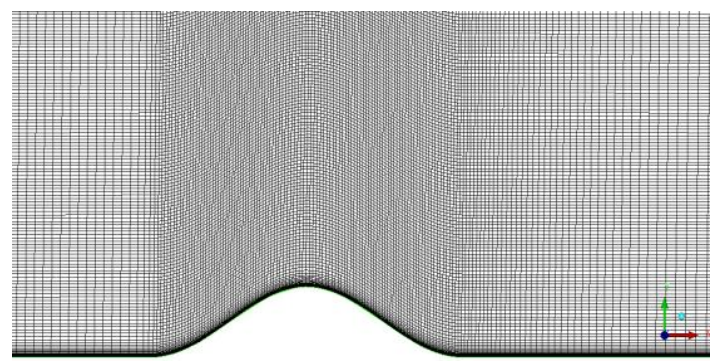

Figure 3. The grid mesh around the hill Schematic.

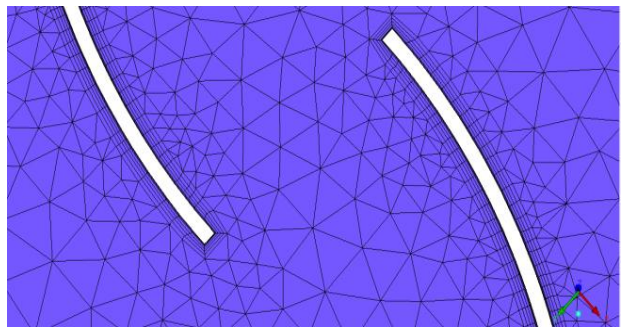

Figure 4. The grid mesh around the blades.

Fig. 5 shows the boundary conditions and the computational domain. The inlet boundary condition is applied on to three faces [8]: the front and sides. The inlet boundary condition uses an atmospheric boundary layer profile as presented in equation (6) [9], with a horizontal velocity of 9 $\mathrm{m} / \mathrm{s}$ value at the height of the hilltop. The atmospheric boundary layer profile is defined as follows:

$U(z)=\frac{u_{*}}{k} \ln \left(\frac{z}{z_{0}}\right)$

where:

$\mathrm{u}_{*}$ : Frictional velocity.

$\mathrm{k}$ : Von Karman coefficient $(\mathrm{k}=0.4)$.

$\mathrm{z}_{0}$ : Roughness parameter $(\mathrm{Z} 0=0.01 \mathrm{~mm})$.

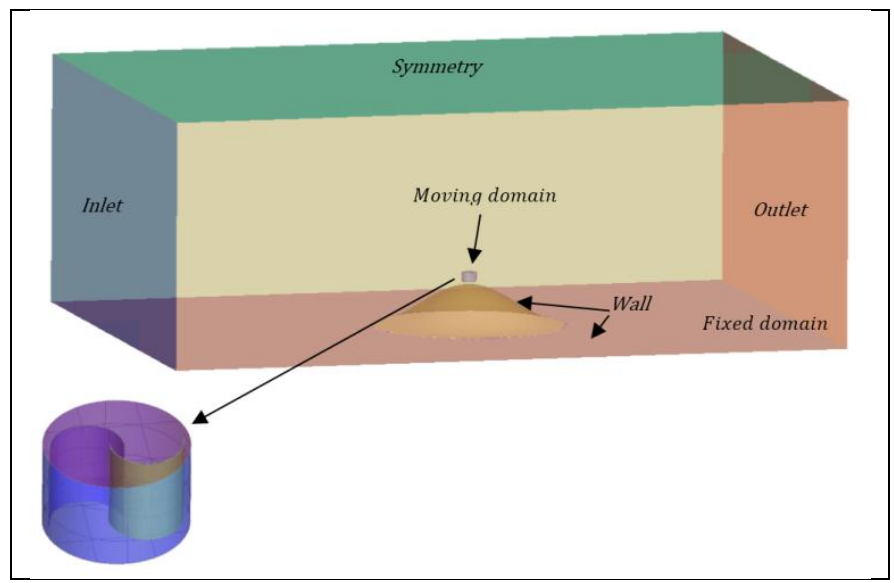

Figure 5. Computational Domain and Boundary Condition.

\section{GRID AND TIME STEP INDEPENDENCE ANALYSIS}

A mesh independence test is conducted in order to select the mesh needed to be in the converged spatial discretization regime for the moment coefficient value. The computational domain meshes are progressively refined until the observed physical quantities are stabilized. The grid refinement is performed using three types of meshes consisting of 1.0, 1.5, and 2 million grids. The convergence of each case needed more than 6 cycles. Fig. 6 shows the moment coefficient during the last two rotations of the conventional turbine rotor versus the total number of cells used. The computed moment coefficient does not show a significant difference when the total number of cells used is more than 1 million. The difference in moment coefficients between the grids of 1.0 and 1.5 million is in the order of $4 \%$, and this difference decrease to $0.4 \%$ between grid of 1.5 and 2 million grids. Based on these results, the following computations were carried out using a mesh consisting of 1.5 million cells.

Several studies also present a temporal discretization analysis: [10] uses 1 degrees/step, [11] reports 0.25 degree/step, while [12] uses 0.12 degrees/step, and [13] uses $0.02 \mathrm{~s}$. Based in these results, a verification study was performed using time steps ranging from 0.5 degrees/step to 1.5 degrees/step at a tip speed ratio of 1.0 and a free stream speed of $9 \mathrm{~m} / \mathrm{s}$. Periodic convergence of the rotor torque output was then evaluated by monitoring the dynamic torque for the selected time steps over the last two revolutions. Fig. 6 demonstrates that time step independence is achieved with a time step of 1 degree/step as for 0.5 degrees/step only a slight change in the torque is observed. The maximum difference is $3.13 \%$. Therefore, to reduce the time of calculation, the conservative choice in this study is to select a time step of 1 degree/step.

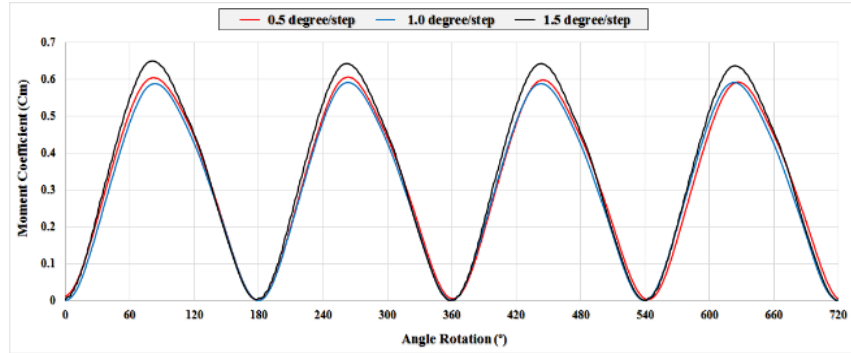

Figure 6. Torque variation during the last two revolutions at $\lambda=1.0$ and 9 $\mathrm{m} / \mathrm{s}$ for different time steps (conventional rotor).

\section{VALIDATIONS OF THE NUMERICAL METHODOLOGY}

In this part, two validations are performed to evaluate the proposed numerical methodology. The simulation results of the flow around a helical wind turbine Savonius with two blades using the SST turbulence model, were compared with the experimental results from [6]. Fig. 7 shows the comparison of the predicted average power coefficients with the measured 
data for various tip speed ratios (TSRs). A maximum power coefficient for conventional rotor $C_{P \max }$ of 0.155 is computed for the tip speed ratio of 0.8 , and a maximum power coefficient for the helical rotor $C_{P \max }$ of 0.169 was found at around 0.70 tip speed ratio. The computational results have a good agreement with experimental data for both cases.

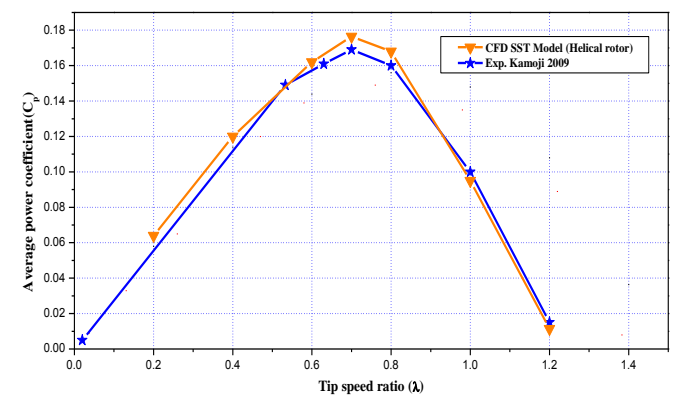

Figure 7. Comparison of the power coefficients from numerical and experimental results.

The second validation is related to the numerical simulation of the flow over the three-dimensional hill. The experimental data is obtained from a wind tunnel investigation by [13]. Fig. 10 shows the good agreement of the vertical profiles of the normalized velocity component of $(\mathrm{U})$ at various positions on the centerline of the hill.

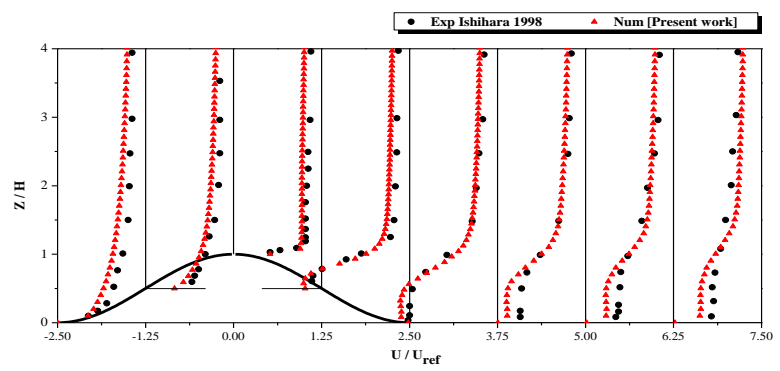

Figure 8. Comparison of the vertical profiles of mean velocity component ( $U$ $\left./ U_{\text {ref }}\right)$.

\section{RESULTS AND DISCUSSION}

\section{A. $\quad$ Effect of different height positions $(L)$}

Numerical simulations of the flow over the hill mounted wind turbine were conducted for different placement height $\mathrm{L}$ : $\mathrm{L}=0.25 \mathrm{~m}, \mathrm{~L}=0.5 \mathrm{~m}$ and $\mathrm{L}=1 \mathrm{~m}$ above the hill. The results of the average power coefficient extracted from both wind turbines, as a function of the tip speed ratio, for a wind velocity inlet of $9 \mathrm{~m} / \mathrm{s}$ are presented in Fig. 9.

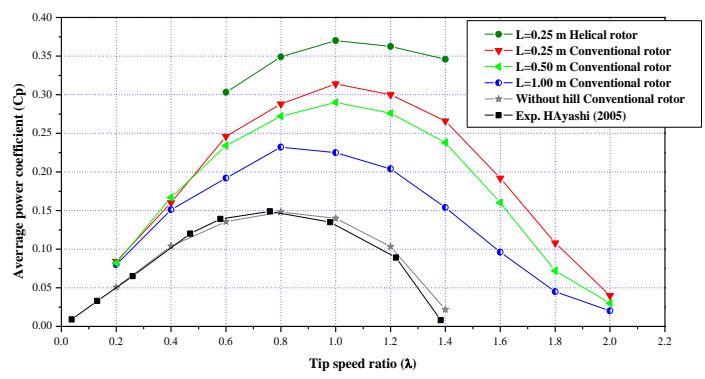

Figure 9. Average power coefficient at different vertical locations of the rotor.

In order to evaluate the power coefficient obtained from an isolated wind turbine both experimental and numerical cases are also reported. One can observe that the maximum values of the average power coefficient is obtained at a height of $\mathrm{L}=0.25$ $\mathrm{m}$ from the top of the hill. The position closet to the hill provides the higher power coefficient as the velocity is highest when measured close to the surface of the hill. The increase in maximum $\mathrm{Cp}$ is more than double than for an undisturbed flow. The maximum also occurs at a higher TSR as the effective wind reaching the turbine is higher. In fact, the ratio of maximum velocities squared $(12.372 / 92=1.89)$ is almost double and is close to the observed $\mathrm{Cp}$ increase. Taking this best position ( $\mathrm{L}=0.25$ ) obtained for the conventional rotor, and calculating the $\mathrm{Cp}$ for the vertical-axis helical wind rotor gives an even higher maximum $\mathrm{Cp}$. The maximum value of power coefficient for helical rotor is approximately 0.37 while, it is 0.32 for the conventional rotor in the tip speed ratio of 1 . This increase in power coefficient is due to the generation of swirling flow generated inside the wind turbine, these observations are also reported by [6] and [14]. This increase is also about twice the difference between the two turbines in an unperturbed flow.

\section{B. Effect of hill height}

In this section, the effect of the hill size on the power production is analyzed for the best position of the turbine rotor found previously. Numerical simulations were performed for the turbine position at $\mathrm{L}=0.25 \mathrm{~m}$ for different hill heights. Three cases are studied: hill height $=1 \mathrm{H}$, hill height $=2 \mathrm{H}$ and hill height $=3 \mathrm{H}$. Note that $\mathrm{H}$ is the standard height of the hill used in the previous section. The atmospheric boundary layer is set such that it has $9 \mathrm{~m} / \mathrm{s}$ velocity at the respective height, i.e. $\mathrm{H}, 2 \mathrm{H}$ or $3 \mathrm{H}$. A very important increase of the power coefficient was obtained, as shown in Fig. 10. 


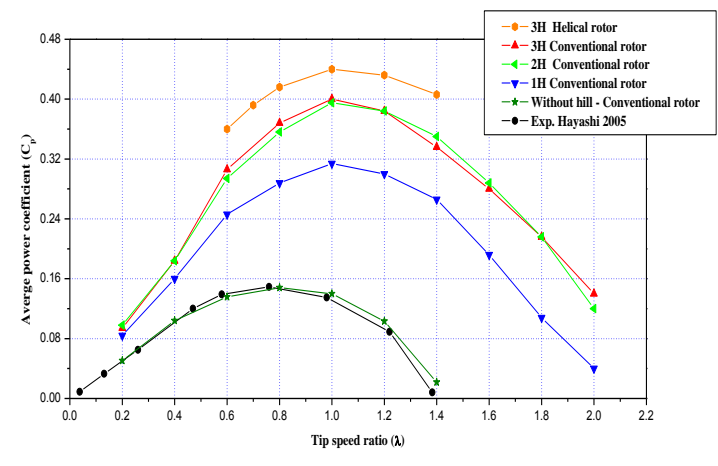

Figure 10. Variation of average power coefficient at different hill heights.

This figure illustrates the influence of the hill height on the performances up to a certain height. The cases of hill height $=2 \mathrm{H}$ and $3 \mathrm{H}$ give approximately the same maximum power coefficient which is close to 0.4 . The same boundary conditions and the hill height are applied to simulate the helical rotor. Again, the power coefficient of this newer design is higher than that obtained by the conventional rotor at the same conditions. A $\mathrm{Cp}=0.44$ is reached which is very high for a Savonius turbine. The difference between the three cases is quite clear in Fig. 11; the velocity in the case of $1 \mathrm{H}$ arrives at $18 \mathrm{~m} / \mathrm{s}$, while it is reaches $25 \mathrm{~m} / \mathrm{s}$ in the case of $2 \mathrm{H}$ and $3 \mathrm{H}$, which explain that the velocity is growing by $25 \%$ when the height is doubled.

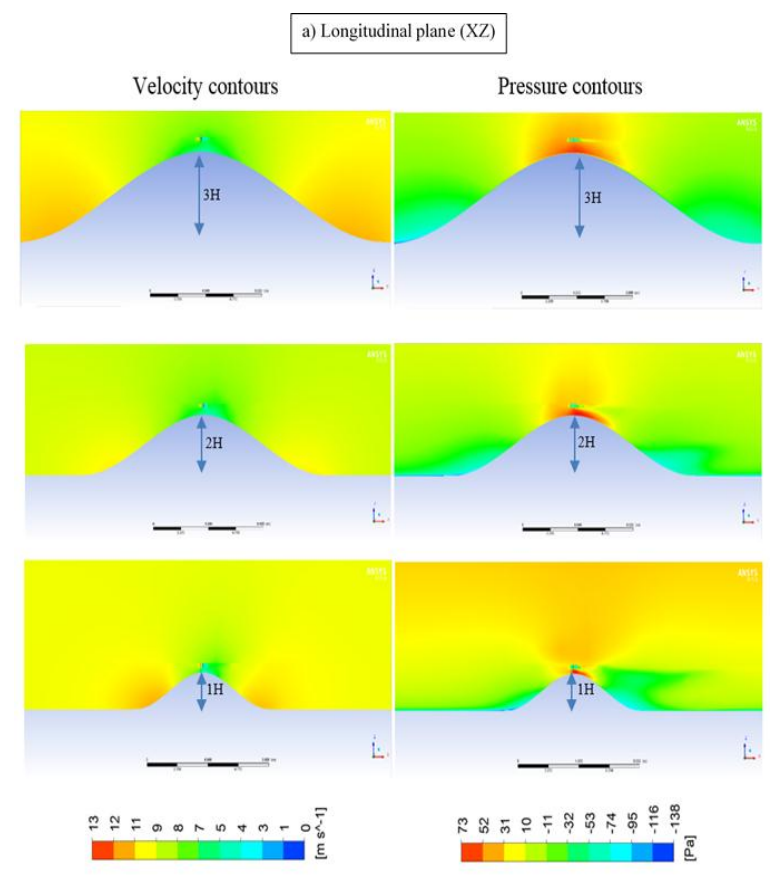

Figure 11. Velocity and pressure contours at different plane with different hill heights (Conventional rotor). a) Longitudinal plan (XZ), b) Transversal plan (YZ).

These planes are vertical to the rotor axis to display the turbulent features around the helical rotor as indicated in

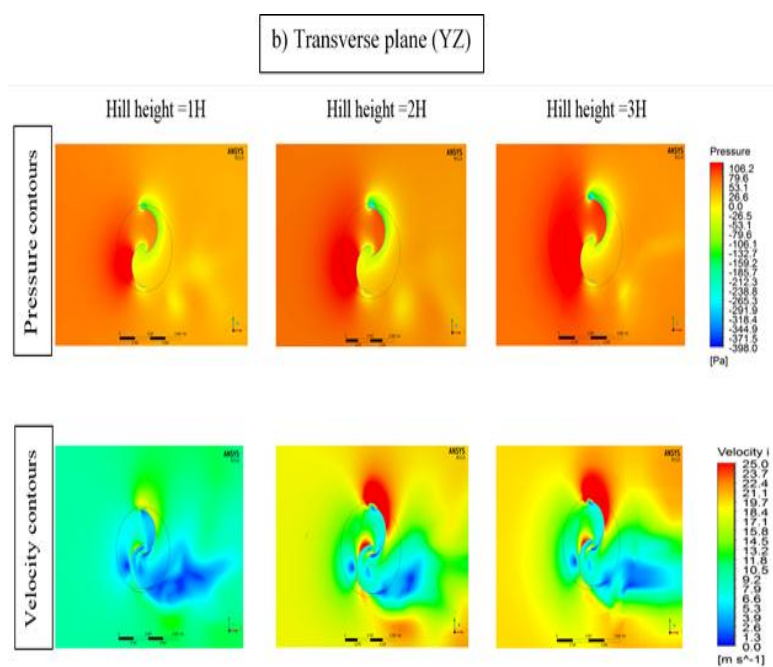

Fig.12. The pressure distribution and contours of the velocity at the maximum power coefficient are presented and compared in Fig. 13 for different sections. The flow fields at each horizontal section are not identical due to its helical geometry along the rotor axis. At this angle, plan 4 and 5 report that this part of the blade contributes significantly to the creation of clockwise torque. These results show that the wind did not directly influence the concave surface of the blade. Instead, it impacted on the convex surface of blade 2, and then hit the concave surface of blade 1

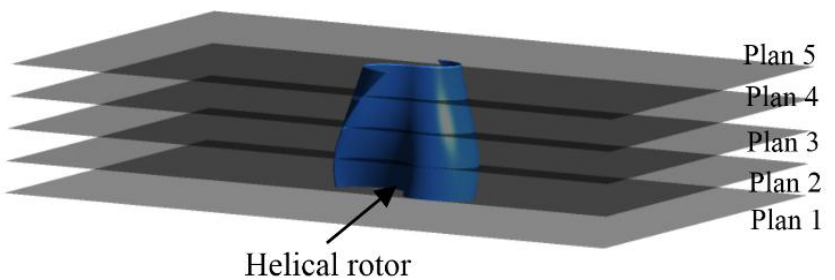

Figure 12. Fig. 18. Vertical sections of blades.

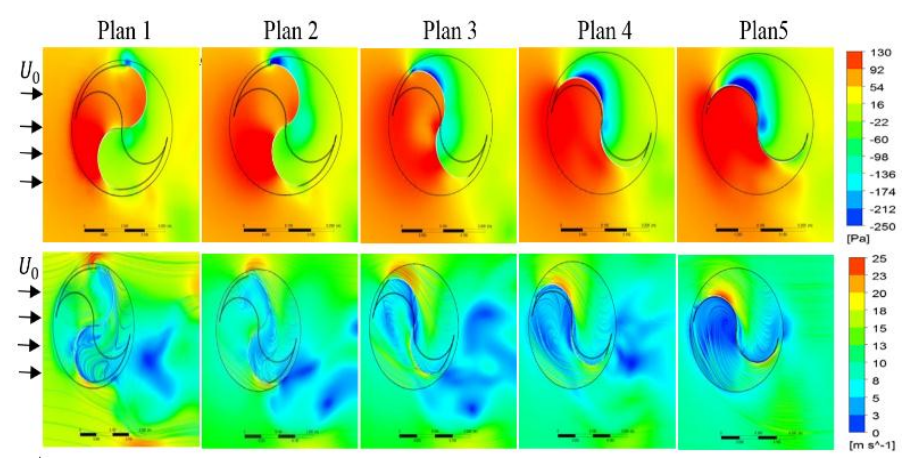

Figure 13. Velocity and pressure contours at different section vertical of helical rotor.

\section{Influence of wind velocity}

The main factor affecting the power output of wind turbine is the incoming wind velocity. The importance of wind speed is that the power is directly proportional to the cube of wind speed according to equation 2 . The power coefficient 
represents the aerodynamic efficiency of the wind turbine, which differs from one turbine to another. Three simulations are performed for different incoming wind velocities using the same computational domain and boundaries conditions as well as the best position from the previous cases $(\mathrm{L}=0.25 \mathrm{~m}$ and hill height $=3 \mathrm{H}$ ). The objective is to analyze the effect of the velocity on the coefficient of power.

Fig. 14 shows the numerical results of the average power coefficients at different Reynolds numbers calculated by equation (5) for three speeds $(6,9,12,15 \mathrm{~m} / \mathrm{s})$. The difference between the three cases is clearly illustrated in this figure, there is a proportional relation between wind velocity and power coefficient, it can be noted that the average power coefficient increases slightly with increasing wind velocity. The maximum value of the power coefficient appears in the range of the tip speed ratio of $(\lambda=0.8$ to $\lambda=1.2)$, which agree with the previous studies [5].

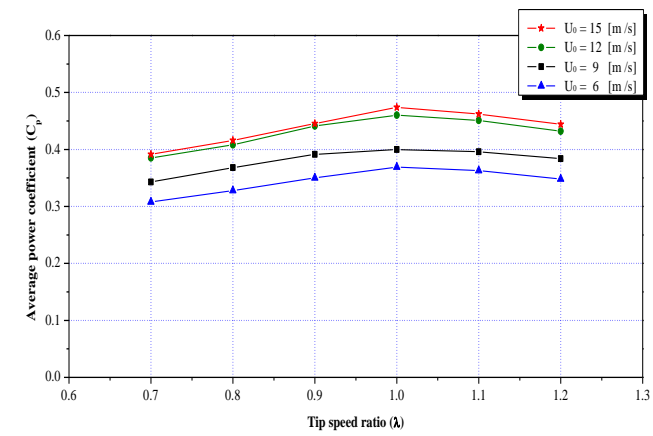

Figure 14. Power coefficient as a function of tip speed ratio at different wind velocity.

\section{CONCLUSION}

In this study, the flow analysis over a three-dimensional hill with a one-stage Savonius turbine is carried out numerically. Results obtained by the SST turbulence model are analyzed and compared with experimental data. The proposed methodology indicates that the numerical predictions of the flow field as well as power coefficient calculations are in reasonable agreement with experiment data. The flow around a three dimensional hill is characterized by an acceleration of the wind towards the hilltop and deceleration on the leeward side. It is demonstrated that it is possible to improve the low performance of the Savonius turbine by placing them at the top of a hill, which is clearly the best position. The extracted power increases significantly, more than doubles, when placed on top of a hill. The study of the aspect ratio of the Savonius turbine indicates that the optimum aspect ratio is different when the turbine is placed on the hill. The paper also shows that a Savonius rotor with a helical geometry always performs better than the conventional Savonius turbine.

\section{REFERENCES}

[1] Kim, H.-G. and V. Patel (2000). "Test of turbulence models for wind flow over terrain with separation and recirculation." Boundary-Layer Meteorology 94(1): 5-21.

[2] Castro, F. A., et al. (2003). "Simulation of the Askervein Flow. Part 1: Reynolds Averaged Navier-Stokes Equations (k_E Turbulence Model)." Boundary-Layer Meteorology 107(3): 501-530.

[3] Sørensen, N. N. (1995). General-purpose flow solver applied to flow over hills, Technical University of Denmark Danmarks Tekniske Universitet, Ris $\emptyset$ National Laboratory for Sustainable EnergyRis $\emptyset$ Nationallaboratoriet for Bæredygtig Energi, Wind Energy DivisionAfdelingen for Vindenergi, Aeroelastic DesignAeroelastisk Design.

[4] Lun, Y. F., et al. (2003). "Numerical simulation of flow over topographic features by revised $\mathrm{k}-\varepsilon$ models." Journal of Wind Engineering and Industrial Aerodynamics 91(1): 231-245.

[5] Hayashi, T., et al. (2005). "Wind tunnel tests on a different phase threestage Savonius rotor." JSME International Journal Series B Fluids and Thermal Engineering 48(1): 9-16.

[6] Kamoji, M., et al. (2009). "Performance tests on helical Savonius rotors." Renewable Energy 34(3): 521-529.

[7] Liu, Z., et al. (2016). "LES study of turbulent flow fields over a smooth 3-D hill and a smooth 2-D ridge." Journal of Wind Engineering and Industrial Aerodynamics 153: 1-1.

[8] Larin, P., et al. (2016). "CFD based synergistic analysis of wind turbines for roof mounted integration." Journal of Wind Engineering and Industrial Aerodynamics 156: 1-13.

[9] Ishihara, T. and K. Hibi (2002). "Numerical study of turbulent wake flow behind a three-dimensional steep hill." Wind and Structures 5(2_3_4): 317-328.

[10] Shaheen, M., et al. (2015). "Numerical study of two-bucket Savonius wind turbine cluster." Journal of Wind Engineering and Industrial Aerodynamics 137: 78-89.

[11] Mc Tavish, S., et al. (2012). "Steady and rotating computational fluid dynamics simulations of a novel vertical axis wind turbine for smallscale power generation." Renewable Energy 41: 171-179.

[12] Wang, Y.-F. and M.-S. Zhan (2015). "Effect of barchan dune guide blades on the performance of a lotus-shaped micro-wind turbine." Journal of Wind Engineering and Industrial Aerodynamics 136: 34-43.

[13] Krishnan, A. and M. Paraschivoiu (2015). "3D analysis of building mounted VAWT with diffuser shaped shroud." Sustainable Cities and Society.

[14] Ishihara, T. and K. Hibi (1998). An experimental study of turbulent boundary layer over a steep hill. Proceedings of the 15th National Symposium on Wind Engineering.

[15] Akwa, J. V., et al. (2012). "Discussion on the verification of the overlap ratio influence on performance coefficients of a Savonius wind rotor using computational fluid dynamics." Renewable Energy 38(1): 141149. 